\title{
Sexual and Reproductive Health Sexual and Reproductive Health Knowledge, Attitude and Practice SURVEY and Service Quality Assessment in Tirana
}

\author{
Miranda Spahiu, PhD Candidate \\ Eris Dhamo, PhD \\ Elda Hallkaj, PhD \\ University of Tirana, Social Science Faculty, Albania
}

URL:http://dx.doi.org/10.19044/esj.2016.v12n20p1

\begin{abstract}
Sexual and reproductive health services in Albania are based on a positive history of services quality and access. However, in the context of the past decades changes in the social and population perspective, these services are faced with the challenge to meet increasing and evolving demands from girls and women. The data available are scare to portray a good picture of the knowledge, attitude practice and other dimensions of access and quality of sexual and reproductive services from the viewpoint of girls and women. The findings introduced in this article present an attempt to add on the evidence, with the target of Tirana, being the most populated city. The methodology used in this research is composed by two components: the quantitative KAP survey, and the qualitative assessment of the services' access and quality. The first instrument is tested and has passed the necessary reliability and validity tests and the second instrument is a focus group with girls and women. Both data are analysed with respective software. The respondents reported low level of knowledge on contraception methods, risk factors for cervical cancer, and signs of breast cancer. They accept that practices in terms of modern contraception are not frequent, similar are the prevention measures or testing for cervical cancer, IST/HIV. The study identifies that many barriers remain strong for girls and women in Tirana to access quality services.
\end{abstract}

Keywords: Sexual health, reproductive health, service access and quality 


\section{Introduction}

Albania has a serious commitment for Sexual and Reproductive Health (SRH), as shown by several legal documents and regulations, which are developed. The organization of the health system in Albania is favorable in providing such services, considering that primary health care (PHC) reaches the whole population through the health centers. Traditionally, the utilization of PHC services for antenatal care has been significant, creating opportunities to address SRH issues (MoH, 2013). As result of a strong political will, the health system is under reform, health insurance has been introduced and private health care is developing quickly. International support has been materialized in financial support as well as significant technical assistance.

As of 1 January 2016, the resident population in Albania is 2,886,026. From 2001 the population of Albania has experienced a negative growth. This decrease in the number of the population has come mainly due to emigration, and the decrease of the number of births (a reduction by 32 per cent). The Total Fertility Rate (TFR) has steadily decreased. In 2001, the TFR was above the substitution level, at 2.31 children for one woman in reproductive age. In 2013, the TFR decreased to 1.76 children for one woman of reproductive age (INSTAT 2015).

The Albanian Demographic and Health Survey 2008-09 (ADHS), although not a recent data source, remains a comprehensive reference for information regarding SRH components. Despite the good knowledge on family planning captured in the survey, only $11 \%$ of married women are using a modern method of contraception, other $59 \%$ basing fertility control on traditional methods. By far the most common method used by currently married women is withdrawal (58 percent), followed by the male condom (4 percent), female sterilization (3 percent) and the pill (2 percent). Women living in rural areas and with lower level of education are less likely to use contraception in general and modern methods in specific. Since 2002, use of modern contraception increased from 8 to $11 \%$ but still remains law compared with other European countries. The most common reasons not to use contraception are fear of side effects and husband's opposition to using contraception. From those using contraception, 53\% get their supplies from public facilities, $40 \%$ from the private sector and $7 \%$ from other sources.

Given the country's very low fertility rate and very low use of modern FP methods, it is unsurprising that Albania has a relatively high abortion ratio. In 2008-2009, Albania had 270 abortions for every 1,000 live births, a level higher than estimates for Western Europe as a whole (230 per 1,000). Although 2011 data show a reduction in Albania's ratio-to 242 abortions per 1,000 live births -this reduction coincides with the increase in private-sector clinics in large cities, like Tirana. Anecdotal evidence suggests 
that the official figures for Albania in recent years are underestimates because they do not take into medical abortion and the ones taking place in private-sector clinics.

The same source (ADHS 2008-2009) confirms that the percentage of young women and men aged 15-24 who both correctly identify ways of preventing the sexual transmission of HIV is 35.9\% of females and $22 \%$ of males interviewed. For both women and men, the likelihood of having a comprehensive knowledge about AIDS is greater for those aged 15-24 that for those aged 25-49. For every age, women are more likely to have a comprehensive knowledge than men. Less education is associated with a lack of knowledge on HIV/AIDS issues. Lower social status affects girls and women HIV/AIDS knowledge. Among men, the most important factor that increases the odds of lack of knowledge is living outside Tirana.

The Health Behaviour in School-Aged Children Study, (IPH, 2009) showed that $14 \%$ of students aged 13-15 years are involved in sexual relations. This data appears consistent with findings from the ADHS 20082009.

Data according to Youth Risky Behaviour Survey (IPH, 2009) show that $22.3 \%$ of boys have had sexual relationships with only one partner only, while $25 \%$ have had 2 or more partners. Although there are no gender disaggregated data for the age group of 15-19 years about contraceptive use, ADHS reports that $41 \%$ of youth (F\&M) 15-24 age use traditional methods. Despite the few and in some cases missing data for STIs in adolescent group, the recent years seems a growing trend of STIs and the prevalence of Chlamydia trachomatis infections in women, showed that age 20 years representing $4.8 \%$ of the total cases (IPH, 2011).

The Knowledge Attitude Practice survey (2008) for sexual and reproductive health among 2072 school youth 14-19 years old, reveals that $72 \%$ of interviewed report having heard at school about sexuality, $84 \%$ have heard about IST/HIV/AIDS. 40\% of interviewed express that they have not been talked about contraception, 80\% of interviewed identify AIDS as IST, meanwhile the other STIs are less recognized. Only 36\% of young people admit that the information received has been sufficient, identifying as the main causes the limited hours on the subjects, and the quality of teaching. Around $1 / 3$ of youth have received information on IST and contraception from peers, and 38\% have received it from teachers. Health personnel have been a resource of information for $18 \%$ of cases. $62 \%$ of the interviewed feel uncomfortable when discussing about SRH. Mostly girls (70\%), feel in difficulties and uncomfortable discussing about SRH. 85\% of the interviewed say that they rarely discuss with parents about $\mathrm{SRH}$, and 90\% identify the school context as the suitable place to learn about SRH. 
Needless to confirm that the data summaries so far does not reflect systematically the current situation/needs of girls and women for SRH services. Therefore, the survey reported in this article was undertaken to fill this gap. It intends to help articulate reccomandations to address the current situation of programs and services in the SRH field.

\section{Methodology}

The current research report presents the findings of the Knowledge Attitude and Practice (KAP) survey in Tirana, and the qualitative assessment of the service access and quality. Knowledge, attitude and practice (KAP) survey are widely used to gather information for planning public health programmes in countries. KAP survey were designed to measure the extent to which an obvious hostility to the idea and organisation of family planning existed among different populations, and to provide information on the knowledge, attitudes, and practices in family planning that could be used for programme purposes the world. (Cleland, 1973, Ratcliffe, 1976). Hence KAP survey established their [lace among the methodologies used to investigate health behaviour, and today they continues to be widely used to gain information on health-seeking practices. (Hausmann-Muela et al. 2003, Manderson and Aaby 1992)

The KAP survey intends to give insight regarding the Knowledge, Attitude and Practices on different Sexual Reproductive Health components among girls and women of reproductive age in Tirana. Data have been collected through face to face interviews with 300 girls and women of reproductive age (15-49 years old). The face to face survey is a prospect of probing beneath the surface to get a bit more depth to the information that is obtain. (Denscombe, M. 2010) For sampling the participants in the study, a two-stage cluster sample approach was utilized. The criteria for sampling the location cluster (health centers) has been the area of the Health Centers (urban and rural). The complete list of the health centers was provided by the Regional Health Authority, dividing the rural and the urban clinics. Taking into consideration the number of interviewers engaged, and the total number of clinics, counting from the first, each third in the list was selected. These were referred as the study sites. Women were randomly drawn from each of the sites by identifying a corner and selecting the fifth girl/women to walk by that point until the desired sample size of participants was reached. A total of 12 public primary health clinics, 6 in urban and 6 in rural areas were targeted.

None of the data files collected contained any names from any of the participants. The data on the computer did not have any links to the person providing the information and were be stored on a password protected database on a password protected computer. The data were backed up, for 
data safety reasons. Data analysis was performed using SPSS version 14 . Descriptive statistical analyses, including frequency distributions, percentages, were used to analyze data from the surveys.

The structured survey instrument is composed of the demographic section, breast and cervical cancer, contraception, IST/HIV/AIDS, Abortion, Domestic Violence, and Communication sections.

The quality assessment intended to understand the perception regarding the quality of sexual and reproductive health services among clients (women of reproductive age 15-49) in Tirana. The qualitative approach has been deemed important due to the nature of the study questions/objective. The qualitative approach helped to understand at the level of knowledge, attitudes, behaviors/experience, and social norms/values by using a small sample rather than a large sample. The qualitative approach investigated the why(s) and how(s) of the questions through the analysis of theme interview, not only the what(s), where(s) and when(s).

In the process of data collection, the number of FGDs carried has been determined both by the number of eligible respondents and by the level of additional new information generated over the course of the research. Therefore, a total of 4 FGDs were conducted.

At the time of the FGD, the facilitator obtained oral consent for participation. No personal or sensitive questions were asked for any participants. The FGD instrument guide contained open-ended, clear, and simple worded questions to facilitate participants' understanding. The facilitator ensured that participants had an opportunity to express their views and opinions in a safe and confidential manner. The thematic analysis approach was used. Using structured summary forms, content analysis was conducted on the notes of the FGDs, observation sheets according to the best qualitative research techniques.

\section{Findings}

\section{KAP Survey}

Approx. 20\% of the participants in the KAP survey belonged to young age (15-24 years old); whereas one third belonged to 25-34 years old and the rest were part of 35-49 years old group, almost two-third of them (70.4\%) lived in urban area and only $29.6 \%$ lived in rural ones; more than half of respondents were married; $20 \%$ of them were single and less than ten percent were engaged or in a relationship. Among uninsured ones, the main reason for not being part of health insurance scheme was lack of time to complete the procedure (23\%); the unemployment status (21\%), and/or lack of information (5\%) where to obtain the health insurance book - the procedure. 


\section{Breast and Cervical Cancer}

As reported at Table 1.1, with respect to the knowledge/beliefs on risk factors that cause cervical cancer, the common beliefs where that having multiple partners (30.1\%) and the presence of an STI (30.7\%) are one of the main reasons that may cause cervical cancer. The second most common opinions where related with Human Papiloma Virus (15.5\%) and the early sexual activity (15.2\%). Having multiple partners or pregnancies, genetic factors and smoking were mentioned by less than $10 \%$ of respondents.

\begin{tabular}{|c|c|}
\hline Risk factors for cervical cancer & Valid Percent \\
\hline Early sexual activity & 15.2 \\
\hline Multiple partners & 30.1 \\
\hline His/r partner has/have had multiple partners & 9.2 \\
\hline HPV & 15.5 \\
\hline STI & 30.7 \\
\hline Smoking & 10.6 \\
\hline Genetic factor (family history) & 28 \\
\hline Multiple pregnancies & 8.9 \\
\hline Low immunity & 6.1 \\
\hline Other & 1.7 \\
\hline
\end{tabular}

Table 1.1 Knowledge on Risk factors for cervical cancer

Less than one-third of the respondents (32.7\%) reported that have carried out this PAP smear test, in comparison with $67.3 \%$ of those who haven't carried out. The reasons why they haven't been tested were mainly related with "lack of disease" $(25.5 \%)$ or lack of time/neglected $(14.5 \%)$ or lack of knowledge about this test (9.2\%).

In order to assess their level of knowledge on early breast cancer symptoms, respondents were asked to express one or some symptoms (multiple choices) they may know about early identification/diagnosis of breast cancer. The most common sign mentioned by a great majority of them mentioned was glandule in breast or axils (83.3\%), followed by breast or nipples pain (30.1\%) and discharge from nipples (17.7\%). Changing of breast measure or edema, and irritation/breast skin lacerations were less frequently mentioned by respondents.
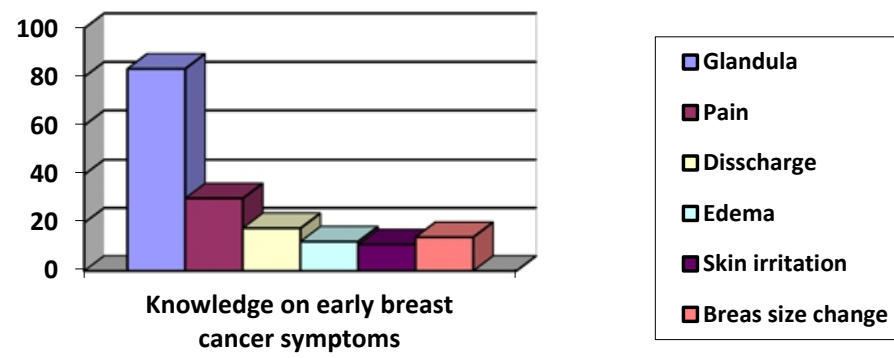

Graphic 1.1 Respondent knowledge on early breast cancer symptoms 
Over than half of respondents (57.7\%) were familiar with techniques how to be self-examined for early symptoms of breast cancer, in comparison with $42.2 \%$ who were not able to self-perform such techniques. Among those who were familiar with these techniques, only $34.5 \%$ perform regularly/monthly breast cancer self-examination.

\section{Contraception}

Knowledge regarding contraception methods condom (89.8\%) and pills (82.8\%) were the most mentioned methods, followed by withdrawal 59.5\%, IUD (37.1\%) and Emergency Contraception Pill (32.9\%). Other methods, such as female/male sterilization, injectable and rhythmic methods were mentioned by less than one-fifth of respondents.

In comparison with the level of knowledge, current use of any family planning methods varied greatly. Less than half of respondents where either not using any family planning method at all (36.3\%) or were relying on withdrawal (40\%) as a mean to avoid unplanned pregnancies. Among modern contraception methods, condom was the most used ones (14.2\%), followed by pills (5\%). Among those who weren't using any family planning method, the main reasons for not using any method were lack of sexual activity (35.8\%) and desire to be pregnant (22.9\%).

When asked about the reason/s why they aren't using any modern family planning method, the most common reason mentioned by almost onethird (32\%) of respondents were comfort ability/happiness with the current method (which is withdrawal). On the other hand, fear of side effects (19.2\%) and modern family planning methods are harmful for the woman health (14.5\%) was other reasons mentioned by respondents.

Nearly half of respondents responded positively about intention to use a modern contraceptive in the future, where $34.3 \%$ mention condom, $10.3 \%$ pills and a few of them mention IUD (1.2\%).

In order to assess the knowledge level of respondents they were asked to express their opinion on how well informed they were about family planning/contraception. Over half of respondents (58.1\%) considered themselves as having good information in regards to family planning/contraception, whereas $12.8 \%$ rated themselves as having very good knowledge. Less than one-third (26.6\%) reported that are less informed about these methods, and only $2.6 \%$ reported to have not all information about FP/contraception.

It is interesting to note that despite the fact how well were informed about FP and contraception, over two-third (79.7\%) were interested to know/learn more about these methods. Whereas less than $20 \%$ were either not interested to learn more (14.7\%) or not sure (5.4\%). 


\section{Knowledge on STI/HIV/AIDS}

Respondents report to be informed about STIs - nearly all of them have heard about HIV/AIDS (96.2); followed by Syphilis (54.1\%), Hepatitis B (32.8\%), and Gonorrhea (30.8\%). To a lesser degree were mentioned Herpes (17.3\%), HPV (12.9\%) and Trichomonas (6\%). Less than half of respondents $(41.6 \%)$ where aware where to perform a STI/HIV test; compared with 58.4\% who were not aware about testing places/institutions. However, when asked about whether have been tested or not for STI/HIV only $8.2 \%$ responded positively to this question. Majority of respondents (81.2\%) perceive themselves as not being at risk for any STI or HIV infection, considering this as the main reason for not performing STI/HIV test. However, more than one third (36.3\%) stated that their knowledge level was low (36.3\%) or had not all the information (3.2\%) about such infectious. Nevertheless, despite their level of knowledge, a great majority of them (81.9\%) would like to receive more information about STI/HIV/AIDS.

\section{Birth and Abortion}

Slightly half of respondents (52.1\%) had children, where 39.6\% had two children, followed by $25.8 \%$ with one child and $14 \%$ with three children. Only $2.3 \%$ reported to have four children. One-third of them mentioned that the last pregnancy was a planned one, compared with 11.7 who admitted that it was an unplanned one.

To assess the prevalence of abortion rates among respondents they were asked whether have had any abortion history. One-fifth (20.6\%) admitted to have had an abortion history and among them, 38.5\% have had only one abortion, followed by $13.7 \%$ of those two times and $2.6 \%$ who have had three or more abortion. Majority of respondents (58.1\%) have carried out abortion in public clinics (maternity), compared with 31\% who reported private clinics as a place where they carried out the last abortion. Medicaments (pills) were reported by $8.5 \%$ of respondents. Among those who reported an abortion history, 52.7\% have received abortion counselling, compared with $34.1 \%$ who haven't been counselled or $12.4 \%$ who don't remember whether have been counselled or not. For majority of them (60.1) decision to interrupt a pregnancy was a joint one (with the partner), followed by personal decision 23.2\%. Health providers decision (7.2\%) or family influences $(1.4 \%)$ were less frequently mentioned by respondents. In regards to reasons for abortion, economic (28.5\%) and no more children (24.8\%) were the common reasons mentioned by participants, followed by not a stable relationship (16.8\%) and medical (15.3\%) reasons.

Receiving contraception counselling after abortion was reported by $46.5 \%$ of respondents, compared with $39.4 \%$ who said that haven't received any counselling after abortion. However, half of them (52.6\%) mentioned 
that are very well informed (9\%) or have good knowledge (43.6\%) about abortion side effects. Nevertheless, $78.5 \%$ of them stated that would like to receive more information about abortion side effects.

\section{Qualitative Assessment}

Participants in the FGDs report that they approach health services (public and NGOs) to receive mainly care, such as antennal, postnatal, child care, gynaecologic visits. It is important to note, for the majority of the prevention services approached the non-public sector.

Participants perceive that women in Tirana have access to different sexual and reproductive health services; however each of them presents barriers. The access to the public health centres, close to their living areas is conditioned by the health booklet (if women are ensured), and limited range of services offered there. The private sector, of course is conditioned by high prices.

Young people in the FGD express the lack of information on sexuality education. They report the failure of the school curriculum to provide with info, and the public health centre staff. When asked about the experiences with the Health centre (HC) staff, they reported that they have 'no connection', that they 'approach the Health centres only when they are seek', and there is no intention to ask them regarding sexuality education.

Apart from the relationship and trust to be built with the young people, the current communication skills of public HC staff are lacking with young people, failing to approach them in language/terminology (e.g. the rights perceptive, age appropriate information) and techniques (small groups, HC staff visiting school regularly), exploring and respecting/taking into account the context. Additionally, young people recognize the need of public HC staff to work with parents and teachers on sexuality education.

Women access and quality to public reproductive health services are triggered by the insurance status and financial barrier to pay the fee. Women participating at the FGDs report a general lack of trust in the trust in the public health staff competences. The public health system organization sometimes hinders the quality of services, for example the fragmented services for pregnant women for urban areas.

Although women report that public health staff is friendly, they lack the communication skills to transmit information in a practical manner, leaving out the medical terminology.

In regard to the participants' characteristics, the findings differ slightly among rural and urban, the rural participants reporting the barriers related to the cultural perception and health care related behaviour. Additionally, younger age women are prone to access HC, when compared 
to older age women, who represent more cultural barriers towards reproductive health issues.

\section{Conclusion}

Based on the findings of two components of the study, a number of implications for actions/interventions are presented to orient stakeholders at different levels

- The need to advocate at policy makers to develop and implement a framework of sexual reproductive health service provision based on rights, owing to the need to target different groups of population and the sensitivity of the issues involved.

- Plan and implement interventions that have a holistic, integrated and comprehensive approach towards sexual and reproductive health rights. This approach needs to be reflected in the governmental regulatory framework, capacity development plans, broad range of topics, indicators and evaluation of services.

- Plan and implement efforts to improve the access of the young people, especially regarding Sexuality Education, by developing and implementing targeted interventions at central and local levels (guidelines, staff capacity development, collaboration among other actors).

- Develop communication strategies to address the needs of different groups of population, young people, men, women, Roma, etc., acknowledging the necessity for tailored interventions.

- Empower the health prevention and promotion component, utilizing innovative and effective interventions, since the traditional ones have not yield results. Improve awareness of all vulnerable groups of their entitlements and rights and of the SRH services available to them.

- Improve efforts to understand the needs of the population for SRH through continues data collection, and as a result develop practice standards to improve service delivery.

\section{References:}

Cleland, J (1973 ). A critique of KAP studies and some suggestions for their improvement. Studies in Family Planning 4.

Denscombe, M. (2010), The Good Research Guide for small-scale social researche projects, Fourth edition, Open University Press, England Hausmann-Muela, S., R. J, Muela and I. Nyamongo 2003. Health -seeking behaviour and the health system's response. DCPP Working Paper no 14. Institute of Statistics, Albania, 2015 
Institute of Statistics of Albania(INSTAT) and Institution of Public Health(IPH), Albanian Demographic and Health Survey, 2008-09

Institute of Statistics of Albania(INSTAT) and Institution of Public Health(IPH), The Knowledge Attitude Practice Survey, 2008

Institute of Public Health, Albania, 2011

Institute of Public Health, The Health Behaviour in School-Aged Children Study, 2009

Institute of Public Health, Youth Risky Behaviour Survey, 2008

Manderson, L. and P. Aaby, 1992. An epidemic in the field. Rapid assessment procedures and health research. Social Sciences and Medicine 35.

Ministry of Health, 2013

Ratcliffe, J. W. 1976. Analyst biases in KAP Surveys: A cross-cultural comparison. Studies in Family Planning 7 